\title{
2D Materials-Coated Plasmonic Structures for SERS Applications
}

\author{
Ming Xia \\ Applied Materials Inc., Santa Clara, CA 95054, USA; xiaming@g.ucla.edu
}

Received: 27 February 2018; Accepted: 10 April 2018; Published: 12 April 2018

\begin{abstract}
Two-dimensional (2D) materials, such as graphene and hexagonal boron nitride, are new kinds of materials that can serve as substrates for surface enhanced Raman spectroscopy (SERS). When combined with traditional metallic plasmonic structures, the hybrid 2D materials/metal SERS platform brings extra benefits, including higher SERS enhancement factors, oxidation protection of the metal surface, and protection of molecules from photo-induced damages. This review paper gives an overview of recent progress in the 2D materials-coated plasmonic structure in SERS application, focusing on the fabrication of the hybrid 2D materials/metal SERS platform and its applications for Raman enhancement.
\end{abstract}

Keywords: surface enhanced Raman spectroscopy; two-dimensional materials; plasmonic structure

\section{Introduction}

Raman spectroscopy is an optical analysis technique providing characteristic spectral information of anlaytes and has a wide variety of applications in chemistry, biology, and medicine [1-4] because of its capability of providing fingerprints of molecule vibration. One major drawback of Raman spectroscopy is the low yield of Raman scattering, leading to weak Raman signals in most cases, and thus ordinary Raman spectroscopy can hardly provide discernable signals of a trace amount of analytes. Surface-enhanced Raman spectroscopy (SERS) makes up this deficiency via plasmon resonance from metallic nanostructures. Molecules adsorbed on the nanostructured metallic surface experience a large amplification of the electromagnetic (EM) field due to local surface plasmon resonance, which leads to an orders of magnitude increase in Raman yield and greatly enhanced Raman signal. SERS is capable of ultra-sensitive detection (single molecule detection) and allows for label-free detection with a high degree of specificity [5-9]. To achieve high SERS enhancement factors, many efforts have been devoted to developing various metallic (mainly Au and Ag) nanostructures to enhance the local EM field [10-16]. In addition to the bare metal SERS structure, a hybrid structure comprised of metal/inorganic materials [14,17-20] is also employed as a SERS sensor.

Two-dimensional (2D) materials, such as graphene and hexagonal boron nitride (h-BN), have unique electronic and optical properties, and attract widespread interest in potential applications in electronic devices, sensors, and energy generation [21-25]. In addition, 2D materials have been explored to enhance Raman signals [26-32]. Since the discovery of graphene's Raman enhancement capability [33], extensive research has been conducted to reveal the enhancing mechanism of two-dimensional materials, as well as their application in Raman enhancement substrates [11,13,30,34-36]. Unlike traditional SERS substrates, 2D materials provide a non-metallic surface with which to enhance the Raman signal. Recently, combining 2D materials with metallic plasmonic structures to form a hybrid SERS platform has become an emerging research field. The 2D materials-coated SERS platform offers synergetic Raman enhancement from both 2D materials and plasmon resonance, and additional advantages such as protection of metal from oxidation and protection of molecules from photo-induced 
damages. This paper will first give a brief introduction of the Raman enhancing mechanism of 2D materials and then discuss the recent process of 2D materials-coated plasmonic structures for SERS application, including their fabrication, sensitivity, and stability.

\section{Raman Enhancement of 2D Materials}

This section will briefly introduce the Raman enhancement mechanism of 2D materials, including graphene, $\mathrm{h}-\mathrm{BN}$, and molybdenum disulfide $\left(\mathrm{MoS}_{2}\right)$. Unlike EM enhancement mechanism of most metallic SERS substrates, Raman enhancement of 2D materials is due to chemical enhancement mechanism $[26,33,37,38]$. Chemical enhancement factor on metallic surface is usually low ( 10-100) [39] compared with EM enhancement factor $\left(\sim 10^{6}-10^{11}\right)$ [5,40,41]. From a broad perspective, chemical enhancement can be considered as modification of the Raman polarizability tensor of molecule upon its adsorption, which in turn enhances or quenches Raman signals of vibrational modes [42,43]. In normal Raman scattering process, molecules are excited by external light to a high-energy level (an intermediate virtual state), and then molecules relax to ground state and emit Raman scattered photons. If the energy of the intermediate virtual state happens to be the same as one of the real electronic levels in the molecule, this scattering process is called resonance Raman scattering, which will have higher scattering efficiency and enhanced Raman signal. Charge-transfer model is often employed to explain chemical enhancement mechanism when molecules are adsorbed on metallic surface. One scenario of charge-transfer mechanisms is that molecules and metal form a surface complex by chemical bonding, which may cause a substantial change in the intrinsic polarizability of the molecule. This new surface complex creates a new electronic state, which is in resonance with the laser and shows enhanced Raman signal. This charge transfer model is the so-called excited state charge transfer model [44]. 2D materials provide a superior platform to study the chemical enhancement mechanism, because they have no dangling bonds in vertical direction and have atomically flat surface, and thus offer a pure system for the study of chemical enhancement effect.

Graphene is the first 2D material used to enhance Raman signals of molecules [33]. Raman enhancement of pristine graphene is ascribed to the ground state charge transfer mechanism [28,37] instead of the aforementioned excited state charge transfer mechanism. In ground state charge transfer process, analyte molecules do not form chemical bond with SERS substrate necessarily, and charge transfer happens when the substrate and the molecules are in the ground state. The charge transfer between molecules and graphene is a physical interaction instead of chemical bonding formation, and thus causes minor change in analytes' electronic distribution. Ground-state charge transfer can easily happen between graphene and molecules adsorbed on its surface because of graphene's two unique features: abundant $\pi$ electrons on its surface and continuous energy band. Figure 1a shows the proposed ground state charge-transfer process between a dye molecule and graphene. In this process, the graphene electrons involvement in the Raman scattered process can enhance the electron-phonon coupling and thus induce the enhancement of the Raman signals [37]. It has been found that graphene Raman enhancement is vibration-mode dependent. The vibrational mode involving the lone pair or $\pi$ electrons, which has stronger coupling with graphene, has highest Raman enhancement $[29,45]$. A more in-depth explanation of graphene-based surface enhancement scattering (GERS) has been given in [37].

h-BN and $\mathrm{MoS}_{2}$ are other two kinds of 2D materials with different electronic and optical properties from graphene. $\mathrm{h}-\mathrm{BN}$ is highly polarized and insulating with a large band gap of $5.9 \mathrm{eV}$ [46]. $\mathrm{CuPc}$ molecule Raman signal is found to be enhanced by h-BN substrate. One proposed Raman enhancement mechanism of h-BN is the interface dipole interaction with analyte molecules, which causes symmetry-related perturbation in the CuPc molecule [26]. In addition, the Raman enhancement factor does not depend on the h-BN layer thickness, because the distribution of the intensity is uniform no matter how thick the h-BN flake is. Atomic layer thin $\mathrm{MoS}_{2}$ is semiconductor and also has a polar bond [47]. For $\mathrm{MoS}_{2}$, both the charge transfer and interface dipole interaction contribute to the Raman enhancement, but both contributions are much weaker compared with graphene and 
h-BN, respectively. The Raman enhancement of $\mathrm{MoS}_{2}$ is not as obvious as that of graphene and h-BN, as shown in Figure 1b.
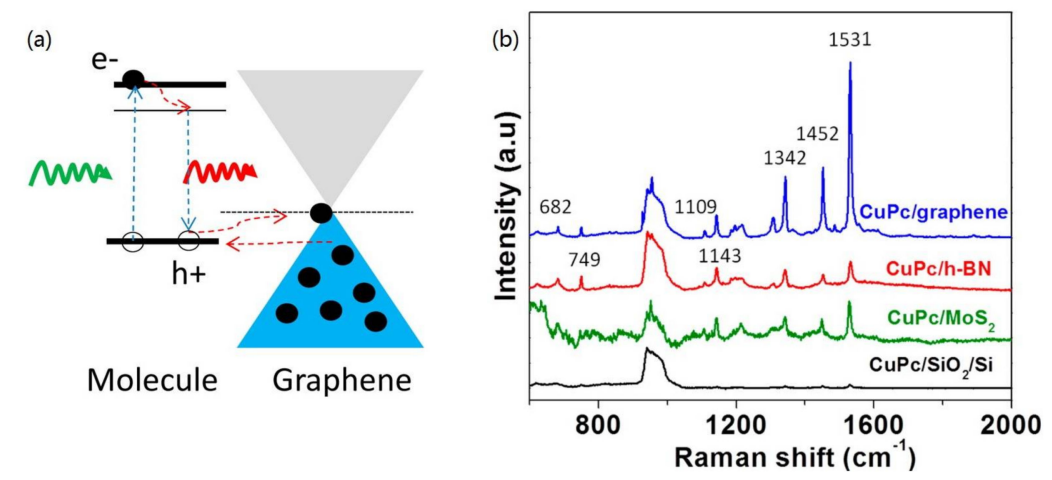

Figure 1. (a) Schematic of the Raman scattered process of graphene-enhanced Raman spectroscopy. Reproduced from [37] with permission; Copyright ACS 2012. (b) Raman spectra of the CuPc molecule on the blank $\mathrm{SiO}_{2} / \mathrm{Si}$ substrate, on graphene, on $\mathrm{h}-\mathrm{BN}$, and on $\mathrm{MoS}_{2}$ substrates. The numbers marked on the peaks are the peak frequencies of the Raman signals from the CuPc molecule. Reproduced from [26] with permission; Copyright ACS 2014.

\section{Two Dimensional Materials-Coated Plasmonic Nanostructures}

Traditional SERS analysis relies on metallic nanostructures that can generate strong local EM field. When combining 2D materials with metallic structure, the hybrid SERS substrate can provide even higher SERS enhancement factor due to the synergic effect of electromagnetic and chemical enhancement. 2D materials, like graphene and h-BN, could offer chemically inert and biocompatible surfaces [48-50], which is favorable in bio-detection. With 2D materials as shielding layer on metallic surface, metallic SERS platforms such as Ag could be protected from oxidation and have longer shelf life, which can improve the stability and repeatability of SERS analysis. The following discussion will focus on the fabrication, sensitivity, and stability of 2D materials/plasmonic structure for SERS application.

\subsection{Fabrication}

2D materials/plasmonic structures require incorporation of 2D materials and metallic plasmonic structures that can provide high local electric field upon laser excitation. Common fabrication methods of 2D materials include mechanical exfoliation, chemical exfoliation, and chemical vapor deposition (CVD). Summary of 2D materials synthesis [51-53] and metallic SERS substrate [54-56] fabrication can be found elsewhere. This section will focus on the incorporation of $2 \mathrm{D}$ materials with metallic plasmonic structure.

One simple way to incorporate 2D materials with metallic nanostructure is to transfer CVD grown 2D materials on metal surface. Graphene and $\mathrm{MoS}_{2}$ have been proven to be capable of overlapping on $\mathrm{Au}$ nanostructures and generating strong Raman signals of graphene and $\mathrm{MoS}_{2}$ [57]. Zhu et al. [58] fabricated graphene-covered gold nanovoid arrays using CVD grown monolayer graphene and investigated the SERS performance of graphene/plasmonic structure. Figure 2 shows the graphene transfer process and the SEM images of graphene-covered gold nanovoid arrays. In this study, graphene was actually suspended on Au nanovoid arrays instead of being conformally coated on Au surface. To achieve 2D material conformally coated SERS substrates, metallic structures need to have certain morphology. For instance, nanopyramid and nanocone structure can be conformally coated with 2D materials, although some ripples are unavoidable. Figure 3 shows graphene-coated $\mathrm{Au}$ nanopyramid [30] and $\mathrm{MoS}_{2}$-coated $\mathrm{SiO}_{2}$ nanocone [59], in which 2D materials are transferred with 
the assistance of poly(methyl methacrylate) (PMMA). Metallic plasmonic structure with conformally coated 2D materials can be better isolated from air and thus has longer stability.
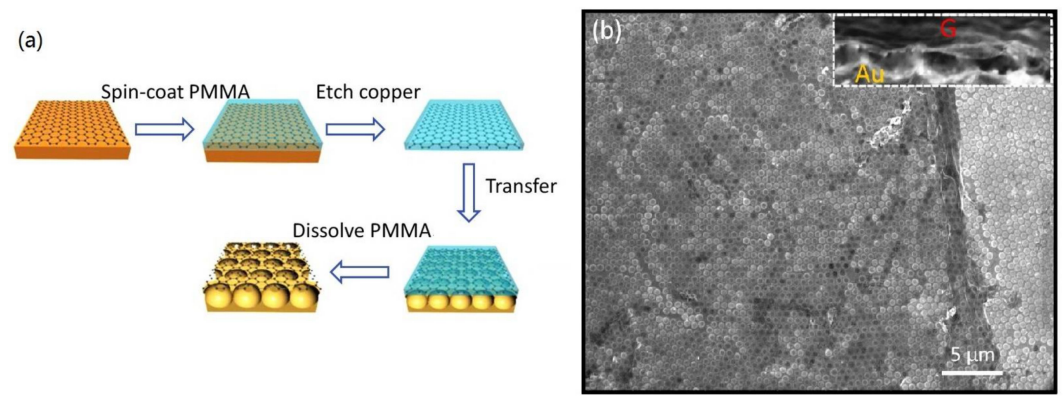

Figure 2. (a) Schematic illustrations of the graphene transfer process. (b) SEM image of a large-area nanovoid array integrated with the transferred monolayer graphene. The dark region is covered by graphene. The inset shows a SEM image of the cross-section of graphene-covered nanovoids. Reproduced from [58] with permission; Copyright ACS 2013.
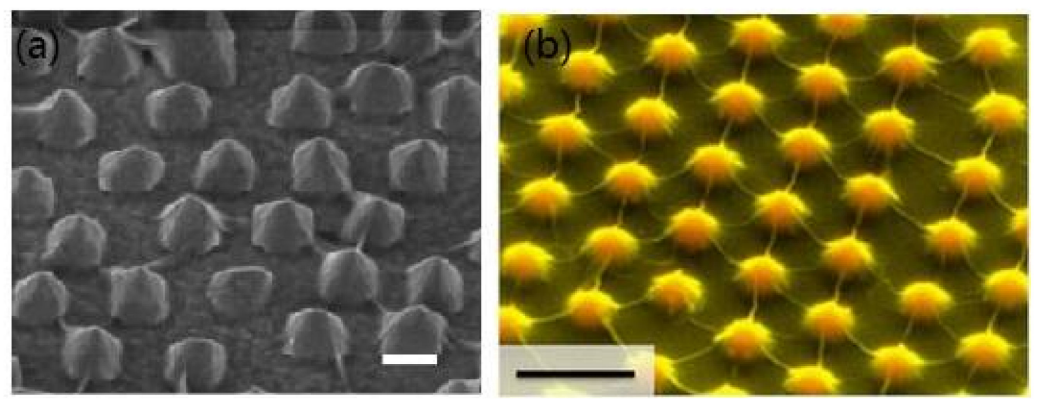

Figure 3. (a) Graphene-coated Au nanopyramid structure. Scale bare is $200 \mathrm{~nm}$. Reproduced from [30] with permission; Copyright ACS 2015. (b) Tilted false-colour SEM image of the 2D strained $\mathrm{MoS}_{2}$ crystal defined by the nanocone array. Scale bar is $500 \mathrm{~nm}$. Reproduced from [59] with permission; Copyright Springer 2015.

PMMA-assisted transfer method has advantage of being able to coat 2D materials for plasmonic structures with various morphologies. However, the drawback is that PMMA residue left on the surface of 2D materials [60] may generate noisy Raman peaks and prevent analyte molecules directly adsorbed on the surface of 2D materials. Therefore, special care needs to be paid to avoid large amount of PMMA residue. Another concern of this transfer method is that the capillary force during the drying process of 2D materials may tear apart the 2D materials and expose the metallic surface to ambient environment. $\mathrm{Xu}$ et al. [27] developed a novel flexible graphene/plasmonic structure with PMMA as a carrying substrate for SERS application. In this study, PMMA was used to support a flat graphene surface instead of a sacrificing transfer layer. Figure 4 shows the fabrication process of the flexible graphene SERS tape.

Another way to incorporate 2D materials with metallic nanostructure is to use chemically exfoliated 2D materials to coat metallic nanoparticles. Kim et al. [61] developed a method with which to sandwich Ag nanoparticles between layers of reduced graphene oxide (rGO) and graphene oxide (GO) in order to prevent Ag nanoparticle from oxidation and boost Raman signals of analytes. Compared with CVD grown 2D materials, chemically exfoliated 2D materials are cost effective and easily to functionalize [61-64]. Figure 5 shows the preparation of SERS substrates with chemically exfoliated graphene. 


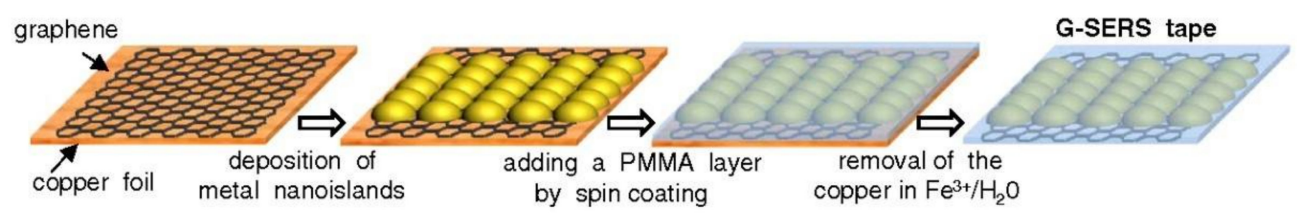

Figure 4. Schematic steps of the preparation route flexible G-SERS tape prepared from CVD-grown monolayer graphene. Reproduced from [27] with permission; Copyright PNAS 2012.

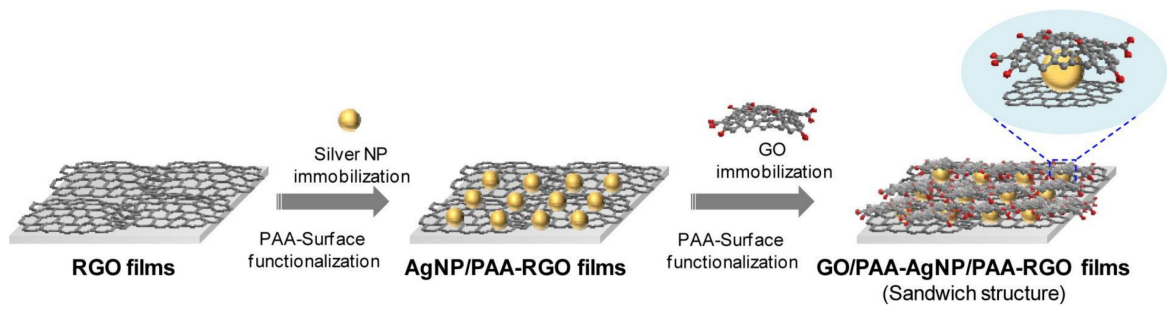

Figure 5. Fabrication Process of GO/PAA-AgNP/PAA-RGO films for application as SERS platform. Reproduced from [61] with permission; Copyright ACS 2012.

Besides ex-situ transferring of 2D materials onto metal surface, in-situ growing 2D materials, like graphene and $\mathrm{MoS}_{2}$, on metal surface is another attractive approach for incorporating 2D materials with plasmonic structure. Liu et al. [65] developed a CVD process to grow graphene shell with controllable thickness on the surface of metal NPs. Figure 6 shows the fabrication process of graphene-encapsulated metal nanoparticles. In situ-grown 2D materials on metal surface do not require 2D materials transfer process and have little chance to have polymer residue left on the surface of 2D materials. CVD in-situ grown 2D materials is a promising method for conformally coating 2D materials on metallic surface. However, due to the high temperature of CVD process, pre-designed metallic nanostructure may change its morphology during high temperature process and lose the pre-designed high local EM field. Low temperature plasma-enhanced CVD method [66-68] could be a potential choice for in-situ growth of 2D materials on metal surface.

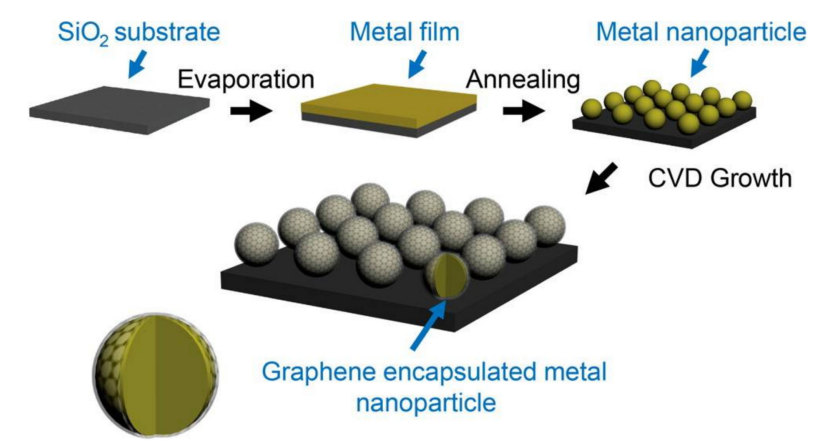

Figure 6. Production process for the Metal@Graphene to serve as a SERS-active substrate. Reproduced from [65] with permission; Copyright ACS 2014.

\subsection{Sensitivity}

Among various 2D materials, graphene is the most widely explored one for incorporation with plasmonic structure. Graphene/metal hybrid SERS platform shows superior SERS performance compared with bare metal SERS substrates. Because of chemical interaction between graphene and target molecules, certain SERS modes are enhanced or prohibited. Although the chemical enhancement factor of 2D materials is not as high as metallic nanostructure, several tens' of times of Raman signal enhancement could be essential when detecting molecules at single molecular level. Several times 
enhancement determines whether the Raman peaks can be seen or not. Comparisons of enhancement factors of different types of SERS sensors are summarized in Table 1. It can be seen that pristine 2D materials generally have lower enhancement factors than metallic SERS substrates or hybrid SERS substrates containing metal. However, a more meaningful comparison between different SERS platforms would require the same analytes and the same detection approach (laser wavelength, laser power, accumulation time, etc.).

Table 1. Comparisons of typical types of SERS substrates.

\begin{tabular}{|c|c|c|c|c|}
\hline SERS Substrate Type & Substrate Materials & Enhancement Factor & Analytes & Ref. \\
\hline \multirow{3}{*}{ Metal } & Au nanotriangles & $1.2 \times 10^{5}$ & Benzenethiol & [69] \\
\hline & Ag nanocubes & $1.25 \times 10^{5}$ & 1,4-benzenedithiol & [70] \\
\hline & Ag nanoparticles & $10^{14}-10^{15}$ & Rhodamine 6G & [71] \\
\hline \multirow{3}{*}{$\begin{array}{l}\text { Metal/inorganic } \\
\text { hybrid structure }\end{array}$} & $\begin{array}{l}\mathrm{SiO}_{2} \text {-coated silver } \\
\text { nanocubes }\end{array}$ & $1.2 \times 10^{6}$ & Rhodamine 6G & {$[72]$} \\
\hline & $\begin{array}{l}\text { Au nanoparticle-coated } \\
\mathrm{ZnO} \text { nanoneedles }\end{array}$ & $1.2 \times 10^{7}$ & Rhodamine 6G & [19] \\
\hline & Au-coated $\mathrm{ZnO}$ nanorods & $10^{6}$ & Rhodamine 6G & [73] \\
\hline \multirow{3}{*}{$\begin{array}{l}\text { 2D materials/Metal } \\
\text { hybrid structure }\end{array}$} & $\begin{array}{c}\text { Monolayer } \\
\text { graphene-coated Au } \\
\text { nanopyramids }\end{array}$ & $10^{10}$ & $\begin{array}{l}\text { Rhodamine } 6 \mathrm{G} \text { and } \\
\text { lysozyme }\end{array}$ & [29] \\
\hline & $\begin{array}{c}\text { Monolayer } \\
\text { graphene-coated Au } \\
\text { nanovoids }\end{array}$ & $10^{3}$ & Rhodamine 6G & {$[58]$} \\
\hline & $\begin{array}{c}\text { Few layer graphene-coated } \\
\text { Au nanoparticles }\end{array}$ & $9.2-19.4$ & Cobalt phthalocyanine & {$[65]$} \\
\hline \multirow{5}{*}{ 2D materials } & Monolayer graphene & $2-17$ & Phthalocyanine & [33] \\
\hline & Monolayer $\mathrm{MoS}_{2}$ & $5 \times 10^{4}-3.8 \times 10^{5}$ & 4-mercaptopyridine & [31] \\
\hline & h-BN & $6.9-41$ & Copper phthalocyanine & [26] \\
\hline & Monolayer $\mathrm{WSe}_{2}$ & $0.18-4.7$ & Copper phthalocyanine & {$[32]$} \\
\hline & $\begin{array}{l}\text { Monolayer graphene on } \\
\text { top of monolayer } \mathrm{WS}_{2}\end{array}$ & $3.8-78.2$ & Copper phthalocyanine & {$[32]$} \\
\hline
\end{tabular}

Wang et al. [29] developed a graphene/Au nanopyramid hybrid SERS platform, which shows single-molecule detection capability for analytes like R6G and lysozyme. In this study, SERS performance of graphene/Au nanopyramid hybrid substrate and bare Au nanopyramid substrate were compared. Table 2 [29] summarizes the average SERS signal enhancement of graphene/Au hybrid substrates over bare Au SERS substrate. As shown in Table 2, additional graphene layer contributes extra few times of Raman enhancement for R6G peak intensity compared with bare Au nanpyramid substrates, which demonstrates the synergic effect of electromagnetic and chemical enhancement from graphene. Even for molecules with small Raman cross-section, like dopamine and serotonin, graphene/Au hybrid platform can still achieve detection limit of $10^{-9} \mathrm{M}$ in simulated body fluid [30]. With graphene/Au nanopyramid hybrid SERS substrates, serotonin molecule Raman peak hot spots and graphene peak hot spots actually coincide, as seen from the Raman intensity mapping of analytes peak with that of the graphene $G$ peak (Figure 7). The results indicate that the intrinsic Raman signal of 2D materials in 2D materials / metal hybrid SERS platform can serve as a gauge of the near-field EM-field intensity to locate hot spots. This unique feature of hybrid platform offers an advantage for molecule detection at ultra-low concentrations. Actual hot spots of SERS substrates are rare and random, even for patterned nanostructure. For extremely diluted solution, the spatial coincidence of molecules and hotspots is rare, leading to long time of up to hours spent on searching for measurable signals. With 2D materials' intrinsic Raman peak intensity as a SERS enhancement factor marker, the hot spots of the 2D materials / metal hybrid SERS platform could be located in advance and speed up the later detection of target molecules. For 2D materials used in hybrid SERS platform with patterned metallic SERS nanostructures, graphene is the ideal choice, because graphene only has a few intrinsic 
Raman peaks, and large-area, high quality graphene is easily achievable. In addition, monolayer graphene has only $2.3 \%$ absorption of the incident laser, and its plasmon resonance frequency is the tetra $\mathrm{Hz}$ regime. Therefore, it has little effect on the EM field of metallic SERS substrates.

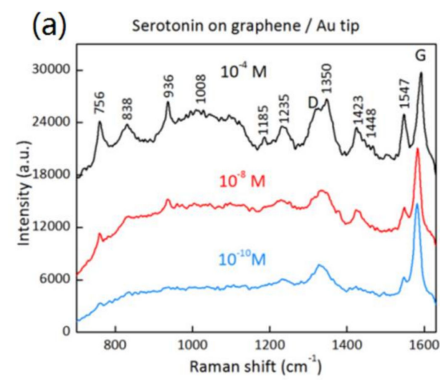

(d)

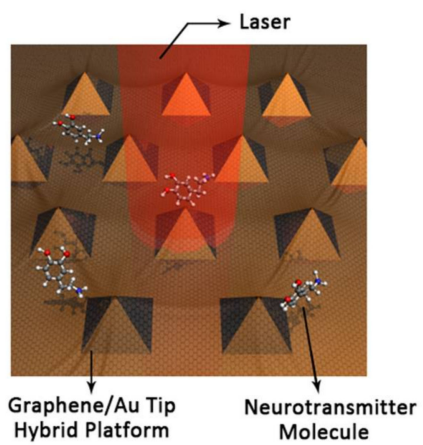

(b)

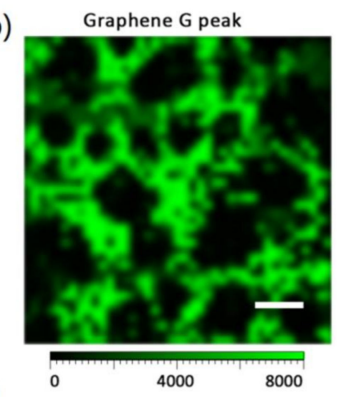

(c)
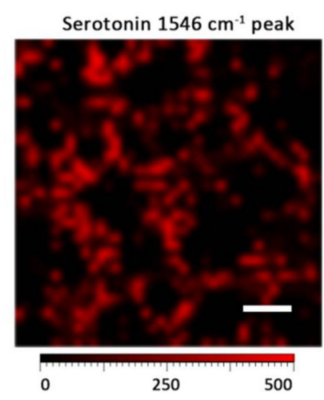

Figure 7. (a) Raman spectra of Serotonin molecules on graphene hybrid structure with 3 different concentrations $\left(10^{-4}, 10^{-8}\right.$, and $\left.10^{-10} \mathrm{M}\right) ;(\mathbf{b}, \mathbf{c})$ Raman intensity mapping of graphene $\mathrm{G}$ band (green) and Raman intensity mapping of serotonin peak at $1546 \mathrm{~cm}^{-1}$ (red) of the same area, scale bar: $10 \mu \mathrm{m}$; (d) Schematic of graphene/Au nanopyramid SERS substrate. Reproduced from [30] with permission; Copyright ACS 2015.

Table 2. Vibration mode dependent enhancement and assignment of Raman peaks in SERS spectra for R6G [29].

\begin{tabular}{ccc}
\hline SERS Peaks $\left(\mathbf{c m}^{-\mathbf{1}}\right)$ & Peak Assignment & Average Enhancement $\left(\boldsymbol{I}_{\text {Graphene/Au tip }} / \boldsymbol{I}_{\text {Au tip }}\right)$ \\
\hline 613 & $\delta(\mathrm{C}-\mathrm{C}-\mathrm{C})$ ip & 10 \\
775 & $\delta(\mathrm{C}-\mathrm{H})$ op & 5 \\
1187 & $\delta(\mathrm{C}-\mathrm{H})$ ip & 2 \\
1311 & $v(\mathrm{C}-\mathrm{C})^{+}, v(\mathrm{C}-\mathrm{N})$ & 6 \\
1360 & $v(\mathrm{C}-\mathrm{C})^{+}, v(\mathrm{C}-\mathrm{N})$ & 6 \\
1506 & $v(\mathrm{C}-\mathrm{C})$ & 4 \\
1577 & $v(\mathrm{C}-\mathrm{O}-\mathrm{C})$ & 8 \\
\hline
\end{tabular}

Note: $I_{\text {Graphene/Au tip }}$ is the R6G Raman intensity obtained on graphene/Au nanopyramid; $I_{\text {Au tip }}$ is the R6G Raman intensity obtained on bare Au nanopyramid.

Besides graphene, h-BN also served as coating layer on plasmonic structure for SERS application. Kim et al. [74] reported h-BN layer-wrapped Au nanoparticles as SERS substrate. h-BN coated Au SERS substrate can provide sensitive detection of aromatic hydrocarbon (PAC) molecules, such as $\mathrm{B}(\alpha) \mathrm{P}$. PAC molecule Raman detection is very difficult using conventional metallic SERS, because the weak interaction between polycyclic aromatic hydrocarbon (PAC) molecules and the metal surface prohibits their adsorption on the metal surface. With h-BN wrapped Au SERS substrates, noticeable and characteristic bands of $\mathrm{B}(\alpha) \mathrm{P}$ can be detected (Figure $8 \mathrm{a}$ ), because the $\pi-\pi$ interaction between $\mathrm{B}(\alpha) \mathrm{P}$ and $\mathrm{h}-\mathrm{BN}$ enlarges the surface adsorption coverage (Figure $8 \mathrm{~b}$ ). 
(a)

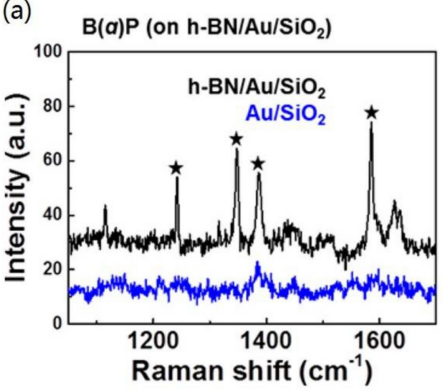

(b)

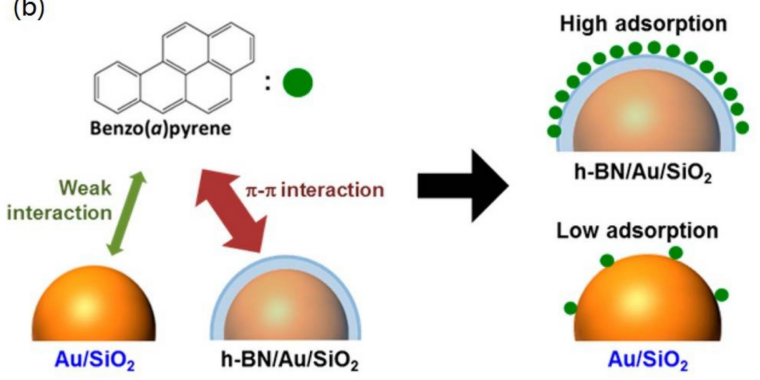

Figure 8. (a) SERS spectra benzo( $\alpha$ )pyrene on $\mathrm{h}-\mathrm{BN} / \mathrm{Au} / \mathrm{SiO}_{2}$ and $\mathrm{Au} / \mathrm{SiO}_{2}$ substrates; (b) schematic mechanism to explain SERS of benzo $(\alpha)$ pyrene on $\mathrm{h}-\mathrm{BN} / \mathrm{Au} / \mathrm{SiO}_{2}$ and $\mathrm{Au} / \mathrm{SiO}_{2}$ substrates. Reproduced from [74] with permission; Copyright ACS 2016.

\subsection{Stability}

Ag nano-structure is known to have excellent SERS performance with wider plasmonic spectral window than other metallic structure made of $\mathrm{Au}$ or Al. However, one major weakness of $\mathrm{Ag}$ nanostructure is that it is easily oxidized in ambient environment. The degradation of Ag will lower the SERS performance and cause uncertainty of analysis. In addition, photo induced damage on analyte molecules is a well know side effect of metallic SERS substrates. This section will discuss recent process of using 2D materials as shielding layer to protect SERS metal substrates from oxidation and protect analyte molecules from photo-induced damages.

2D materials, like graphene and $\mathrm{h}-\mathrm{BN}$, are able to protect metal to be oxidized $[75,76]$. This feature of 2D materials can also be used in SERS substrate development [65,77]. When single layer graphene combines with Ag nanostructure, the hybrid SERS platform provides both better SERS performance and excellent stability in a harsh environment (sulfur) and at high temperatures $\left(300^{\circ} \mathrm{C}\right)$ [78]. Liu et al. [77] combined CVD grown graphene with silver SERS substrates and demonstrated that with the graphene as protecting layer, the hybrid graphene/Ag SERS substrate could achieve large-area uniformity and long-term stability. Li et al. [79] compared the oxidation protection effect between CVD grown graphene and rGO coated Ag nanoparticles. They found out that CVD-grown monolayer graphene served as a better protecting layer than rGO to effectively suppress the oxidation of Ag nanoparticles. As seen from Figure 9, CVD-grown, graphene-coated Ag SERS substrate can provide stable R6G SERS signals up to 28 days with ambient aerobic exposure, while rapidly decreasing Raman signals are seen from rGO-coated and bare Ag nanoparticles. Worse performance of rGO-protected Ag nanoparticle is due to (1) the wide size distribution of rGO results an incoherent thin film and (2) the fact that cracks and holes on rGO film act as a channel to allow air reach Ag surface, leading to the oxidation of Ag nanoparticles. 


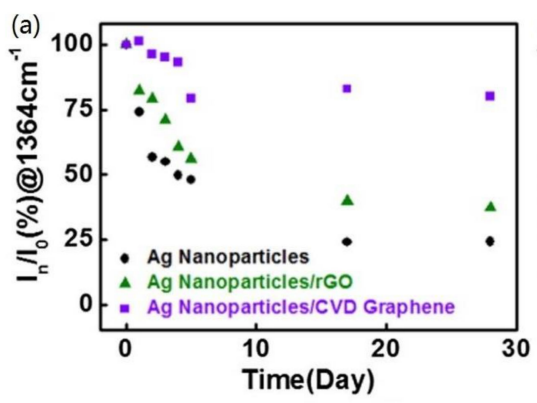

(c)

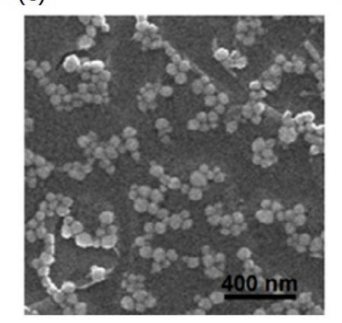

(d)

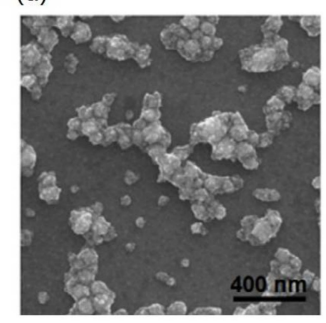

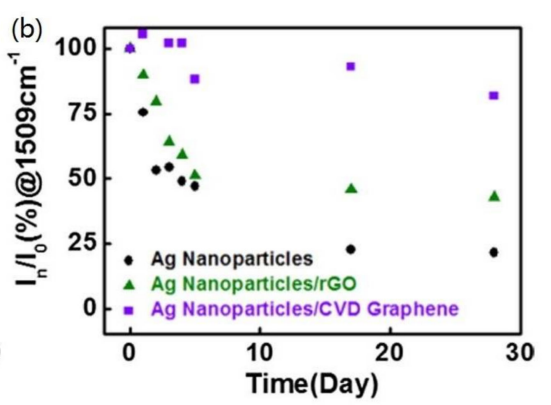

(e)

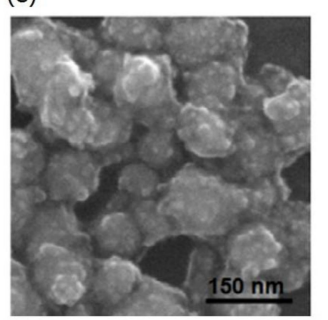

Figure 9. (a) The normalized intensity of the R6G Raman peak at $1364 \mathrm{~cm}^{-1}$ collected using, respectively, the unprotected (black), rGO-protected (green), and CVD graphene-protected (purple) Ag nanoparticles as substrates, versus the time of aerobic exposure; (b) the normalized intensity of the R6G Raman peak at $1509 \mathrm{~cm}^{-1}$ collected using, respectively, the unprotected (black), rGO-protected (green), and CVD graphene-protected (purple) Ag nanoparticles as substrates, versus the time of aerobic exposure; (c) SEM image of the CVD graphene-protected Ag nanoparticles after their 28-day use as the SERS substrate for the measurement of R6G; (d,e) SEM image of the unprotected Ag nanoparticles after their 28-day use as the SERS substrate for the measurement of R6G. Reproduced from [79] with permission; Copyright Elsevier 2013.

Another benefit to combine 2D materials with metallic nanostructures is that 2D materials can help protect molecules from photo-induced damage, such as photobleaching $[27,65,80,81]$. The photobleaching (or photodegradation) of the Raman anlaytes induced by the laser is a well-known side effect in SERS experiments, especially for dye molecules. When combining graphene with metallic nanostructure, the hybrid SERS platform is more stable against photo-induced damage with an even higher enhancement factor. Liu et al. [65] fabricated graphene-encapsulated metal nanoparticles for molecule detection and found out that AuNP/graphene hybrid substrate could significantly suppress photobleaching and fluorescence of cobalt phthalocyanine (CoPc) and R6G molecules. For instance, within the $160 \mathrm{~s}$ measurement period, the $1534 \mathrm{~cm}^{-1}$ peak intensity of CoPc molecules decreases dramatically for Au NPs, while the same peak intensity almost keeps constant for Au@Graphene, as shown in Figure 10a,b. Zhao et al. [81] also demonstrated that graphene can enhance the photostability of R6G molecules with graphene coated Ag SERS substrates during continuous light illumination. Enhanced photostability of molecules provided by graphene during SERS detection is attributed to $\pi-\pi$ interactions between graphene surface and molecules $[65,81]$. Molecule $\pi-\pi$ interaction with graphene allows the charge transfer between graphene and molecules, providing additional path for the molecules to relax from the excitation state to the ground state [82]. This process reduces the number of molecules at excitation states and thus decreases photobleaching rate. Similar protection effect can be achieved by using h-BN layer as well. Kim et al. [74] reported a h-BN film wrapped Au substrate showing extraordinary stability against photothermal and oxidative damages during laser excitation, as shown in Figure 10c,d. This outstanding stability against photothermal damage of h-BN wrapped Au SERS substrate is attributed to the ultrafast heat dissipation through the h-BN layer. With 2D materials as a shielding layer, hybrid SERS substrates will provide long-term stability. 

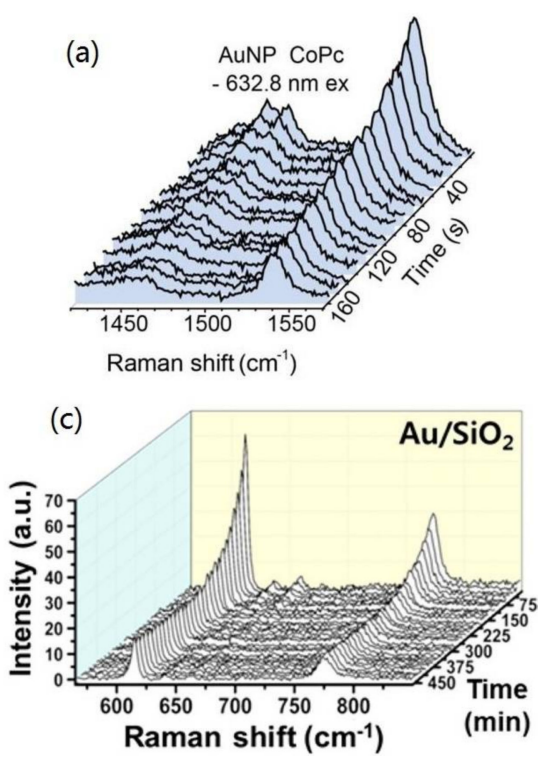
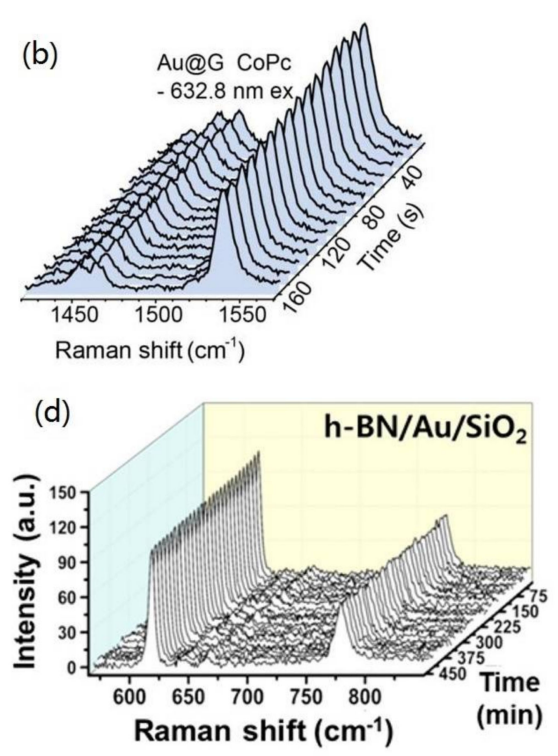

Figure 10. Stability of SERS signals of monolayer CoPc LB films on (a) Au and (b) Au@G. Reproduced from [65] with permission; Copyright ACS 2014. Photothermal and chemical stability of 3 L h-BN/Au/SiO2 substrate; SERS spectra of R6G on the $\mathrm{Au} / \mathrm{SiO} 2$ substrate (c) without and (d) with $\mathrm{h}$-BN protection at different time points (laser power $=0.1 \mathrm{~mW}$, time interval $=15 \mathrm{~min}$ ). Reproduced from [74] with permission; Copyright ACS 2016.

\section{Conclusions and Perspective}

In summary, 2D materials' Raman enhancement is due to the chemical enhancement mechanism, which differentiates them from metallic SERS substrates. Coating 2D materials on metallic SERS substrates introduces extra benefits over bare metal substrates. First, adding 2D materials can further increase the SERS enhancement factor due to the synergic effect of electromagnetic and chemical enhancement. Second, the atomic thin film of 2D materials can help map out the hot spots of the metallic nanostructure without affecting the local EM field of the metallic nanostructure underneath. For example, a Raman mapping of the graphene $G$ peak over the hybrid SERS substrates could give the precise position of the hot spots. Finally, adding 2D materials as a shielding layer offers a chemically inert surface and helps to reduce the fluctuation of the SERS signal caused by the degradation of the metallic nano-structures, photobleaching, or metal-catalyzed site reactions, and thus improves the long-term stability and repeatability of the SERS analysis.

Conflicts of Interest: The authors declare no conflict of interest.

\section{References}

1. Pillai, I.C.; Li, S.; Romay, M.; Lam, L.; Lu, Y.; Huang, J.; Dillard, N.; Zemanova, M.; Rubbi, L.; Wang, Y. Cardiac fibroblasts adopt osteogenic fates and can be targeted to attenuate pathological heart calcification. Cell Stem Cell 2017, 20, 218-232. [CrossRef] [PubMed]

2. Yigit, M.V.; Medarova, Z. In vivo and ex vivo applications of gold nanoparticles for biomedical SERS imagingi. Am. J. Nucl. Med. Mol. Imaging 2012, 2, 232-241. [PubMed]

3. Qian, X.; Peng, X.-H.; Ansari, D.O.; Yin-Goen, Q.; Chen, G.Z.; Shin, D.M.; Yang, L.; Young, A.N.; Wang, M.D.; $\mathrm{Nie}, \mathrm{S}$. In vivo tumor targeting and spectroscopic detection with surface-enhanced Raman nanoparticle tags. Nat. Biotechnol. 2008, 26, 83-90. [CrossRef] [PubMed]

4. Motz, J.T.; Hunter, M.; Galindo, L.H.; Gardecki, J.A.; Kramer, J.R.; Dasari, R.R.; Feld, M.S. Optical fiber probe for biomedical Raman spectroscopy. Appl. Opt. 2004, 43, 542-554. [CrossRef] [PubMed]

5. Le Ru, E.C.; Etchegoin, P.G. Single-molecule surface-enhanced Raman spectroscopy. Ann. Rev. Phys. Chem. 2012, 63, 65-87. [CrossRef] [PubMed] 
6. Luo, S.-C.; Sivashanmugan, K.; Liao, J.-D.; Yao, C.-K.; Peng, H.-C. Nanofabricated SERS-active substrates for single-molecule to virus detection in vitro: A review. Biosens. Bioelectron. 2014, 61, 232-240. [CrossRef] [PubMed]

7. Blackie, E.J.; Ru, E.C.L.; Etchegoin, P.G. Single-molecule surface-enhanced Raman spectroscopy of nonresonant molecules. J. Am. Chem. Soc. 2009, 131, 14466-14472. [CrossRef] [PubMed]

8. Dasary, S.S.; Singh, A.K.; Senapati, D.; Yu, H.; Ray, P.C. Gold nanoparticle based label-free SERS probe for ultrasensitive and selective detection of trinitrotoluene. J. Am. Chem. Soc. 2009, 131, 13806-13812. [CrossRef] [PubMed]

9. Lane, L.A.; Qian, X.; Nie, S. SERS nanoparticles in medicine: From label-free detection to spectroscopic tagging. Chem. Rev. 2015, 115, 10489-10529. [CrossRef] [PubMed]

10. Xia, M.; Zhang, P.; Qiao, K.; Bai, Y.; Xie, Y.-H. Coupling SPP with LSPR for enhanced field confinement: A simulation study. J. Phys. Chem. C 2015, 120, 527-533. [CrossRef]

11. Xia, M.; Zhang, P.; Leung, C.; Xie, Y.H. SERS optical fiber probe with plasmonic end-facet. J. Raman Spectrosc. 2017, 48, 211-216. [CrossRef]

12. Xia, M.; Qiao, K.; Cheng, Z.; Xie, Y.-H. Multiple layered metallic nanostructures for strong surface-enhanced Raman spectroscopy enhancement. Appl. Phys. Express 2016, 9, 065001. [CrossRef]

13. Yan, Z.; Xia, M.; Wang, P.; Zhang, P.; Liang, O.; Xie, Y.-H. Selective manipulation of molecules by electrostatic force and detection of single molecules in aqueous solution. J. Phys. Chem. C 2016, 120, 12765-12772. [CrossRef]

14. Bryche, J.-F.; Bélier, B.; Bartenlian, B.; Barbillon, G. Low-cost SERS substrates composed of hybrid nanoskittles for a highly sensitive sensing of chemical molecules. Sens. Actuators B Chem. 2017, 239, 795-799. [CrossRef]

15. Jimenez de Aberasturi, D.; Serrano-Montes, A.B.; Langer, J.; Henriksen-Lacey, M.; Parak, W.J.; Liz-Marzán, L.M. Surface enhanced Raman scattering encoded gold nanostars for multiplexed cell discrimination. Chem. Mater. 2016, 28, 6779-6790. [CrossRef]

16. Rodriguez-Fernandez, D.; Langer, J.; Henriksen-Lacey, M.; Liz-Marzán, L.M. Hybrid Au-SiO 2 core-satellite colloids as switchable SERS tags. Chem. Mater. 2015, 27, 2540-2545. [CrossRef]

17. Barbillon, G.; Sandana, V.E.; Humbert, C.; Bélier, B.; Rogers, D.J.; Teherani, F.H.; Bove, P.; McClintock, R.; Razeghi, M. Study of au coated $\mathrm{ZnO}$ nanoarrays for surface enhanced Raman scattering chemical sensing. J. Mater. Chem. C 2017, 5, 3528-3535. [CrossRef]

18. Cui, S.; Dai, Z.; Tian, Q.; Liu, J.; Xiao, X.; Jiang, C.; Wu, W.; Roy, V.A. Wetting properties and SERS applications of $\mathrm{ZnO} / \mathrm{Ag}$ nanowire arrays patterned by a screen printing method. J. Mater. Chem. C 2016, 4, 6371-6379. [CrossRef]

19. Liu, L.; Yang, H.; Ren, X.; Tang, J.; Li, Y.; Zhang, X.; Cheng, Z. Au-ZnO hybrid nanoparticles exhibiting strong charge-transfer-induced SERS for recyclable SERS-active substrates. Nanoscale 2015, 7, 5147-5151. [CrossRef] [PubMed]

20. Magno, G.; Bélier, B.; Barbillon, G. Gold thickness impact on the enhancement of SERS detection in low-cost $\mathrm{Au} /$ Si nanosensors. J. Mater. Sci. 2017, 52, 13650-13656. [CrossRef]

21. Zhang, W.; Ma, R.; Chen, Q.; Xia, M.; Ng, J.; Wang, A.; Xie, Y.-H. The electro-mechanical responses of suspended graphene ribbons for electrostatic discharge applications. Appl. Phys. Lett. 2016, 108, 153103. [CrossRef]

22. Chen, Q.; Ma, R.; Lu, F.; Wang, C.; Liu, M.; Wang, A.; Zhang, W.; Xia, M.; Xie, Y.-H.; Cheng, Y. Systematic transient characterization of graphene interconnects for on-chip ESD protection. In Proceedings of the 2016 IEEE International Conference on Reliability Physics Symposium (IRPS), Pasadena, CA, USA, 17-21 April 2016; pp. 3B-6-1-3B-6-5.

23. Zhang, W.; Chen, Q.; Xia, M.; Ma, R.; Lu, F.; Wang, C.; Wang, A.; Xie, Y.-H. Tlp Evaluation of Esd Protection Capability of Graphene Micro-Ribbons for ICS. In Proceedings of the 2015 IEEE 11th International Conference on ASIC (ASICON), Chengdu, China, 3-6 November 2015; pp. 1-4.

24. Xia, F.; Mueller, T.; Lin, Y.-M.; Valdes-Garcia, A.; Avouris, P. Ultrafast graphene photodetector. Nat. Nanotechnol. 2009, 4, 839-843. [CrossRef] [PubMed]

25. Wang, X.; Wang, P.; Wang, J.; Hu, W.; Zhou, X.; Guo, N.; Huang, H.; Sun, S.; Shen, H.; Lin, T. Ultrasensitive and broadband $\mathrm{MoS}_{2}$ photodetector driven by ferroelectrics. Adv. Mater. 2015, 27, 6575-6581. [CrossRef] [PubMed] 
26. Ling, X.; Fang, W.; Lee, Y.-H.; Araujo, P.T.; Zhang, X.; Rodriguez-Nieva, J.F.; Lin, Y.; Zhang, J.; Kong, J.; Dresselhaus, M.S. Raman enhancement effect on two-dimensional layered materials: Graphene, h-BN and $\mathrm{MoS}_{2}$. Nano Lett. 2014, 14, 3033-3040. [CrossRef] [PubMed]

27. Xu, W.; Ling, X.; Xiao, J.; Dresselhaus, M.S.; Kong, J.; Xu, H.; Liu, Z.; Zhang, J. Surface enhanced Raman spectroscopy on a flat graphene surface. Proc. Natl. Acad. Sci. 2012, 109, 9281-9286. [CrossRef] [PubMed]

28. Xu, W.; Mao, N.; Zhang, J. Graphene: A platform for surface-enhanced Raman spectroscopy. Small 2013, 9 , 1206-1224. [CrossRef] [PubMed]

29. Wang, P.; Liang, O.; Zhang, W.; Schroeder, T.; Xie, Y.H. Ultra-sensitive graphene-plasmonic hybrid platform for label-free detection. Adv. Mater. 2013, 25, 4918-4924. [CrossRef] [PubMed]

30. Wang, P.; Xia, M.; Liang, O.; Sun, K.; Cipriano, A.F.; Schroeder, T.; Liu, H.; Xie, Y.-H. Label-free SERS selective detection of dopamine and serotonin using graphene-Au nanopyramid heterostructure. Anal. Chem. 2015, 87, 10255-10261. [CrossRef] [PubMed]

31. Muehlethaler, C.; Considine, C.R.; Menon, V.; Lin, W.-C.; Lee, Y.-H.; Lombardi, J.R. Ultrahigh Raman enhancement on monolayer $\mathrm{MoS}_{2}$. ACS. Photonics 2016, 3, 1164-1169. [CrossRef]

32. Tan, Y.; Ma, L.; Gao, Z.; Chen, M.; Chen, F. Two-dimensional heterostructure as a platform for surfaceenhanced Raman scattering. Nano Lett. 2017, 17, 2621-2626. [CrossRef] [PubMed]

33. Ling, X.; Xie, L.; Fang, Y.; Xu, H.; Zhang, H.; Kong, J.; Dresselhaus, M.S.; Zhang, J.; Liu, Z. Can graphene be used as a substrate for Raman enhancement? Nano Lett. 2009, 10, 553-561. [CrossRef] [PubMed]

34. Xia, M. A review on applications of two-dimensional materials in surface-enhanced Raman spectroscopy. Int. J. Spectrosc. 2018, 2018, 4861472. [CrossRef]

35. Yan, Z.; Xia, M.; Zhang, P.; Xie, Y.H. Self-aligned trapping and detecting molecules using a plasmonic tweezer with an integrated electrostatic cell. Adv. Op. Mater. 2017, 5, 1600329. [CrossRef]

36. Yan, Z.; Liu, Z.; Xia, M.; Efimov, A.; Xie, Y.H. Broadband surface-enhanced coherent anti-stokes Raman spectroscopy with high spectral resolution. J. Raman Spectrosc. 2017, 48, 935-942. [CrossRef]

37. Ling, X.; Moura, L.; Pimenta, M.A.; Zhang, J. Charge-transfer mechanism in graphene-enhanced Raman scattering. J. Phys. Chem. C 2012, 116, 25112-25118. [CrossRef]

38. Ling, X.; Zhang, J. First-layer effect in graphene-enhanced Raman scattering. Small 2010, 6, $2020-2025$. [CrossRef] [PubMed]

39. Liang, E.; Kiefer, W. Chemical effect of SERS with near-infrared excitation. J. Raman Spectrosc. 1996, 27, 879-886. [CrossRef]

40. Kneipp, K.; Wang, Y.; Kneipp, H.; Perelman, L.T.; Itzkan, I.; Dasari, R.R.; Feld, M.S. Single molecule detection using surface-enhanced Raman scattering (SERS). Phys. Rev. Lett. 1997, 78, 1667. [CrossRef]

41. Xu, H.; Aizpurua, J.; Käll, M.; Apell, P. Electromagnetic contributions to single-molecule sensitivity in surface-enhanced Raman scattering. Phys. Rev. E 2000, 62, 4318. [CrossRef]

42. Le Ru, E.C.; Etchegoin, P.G. Principles of Surface-Enhanced Raman Spectroscopy: And Related Plasmonic Effects; Elsevier: New York, NY, USA, 2008.

43. Moskovits, M. Surface-enhanced spectroscopy. Rev. Mod. Phys. 1985, 57, 783. [CrossRef]

44. Adrian, F.J. Charge transfer effects in surface-enhanced Raman scatteringa. J. Chem. Phys. 1982, 77, 5302-5314. [CrossRef]

45. Ling, X.; Wu, J.; Xu, W.; Zhang, J. Probing the effect of molecular orientation on the intensity of chemical enhancement using graphene-enhanced Raman spectroscopy. Small 2012, 8, 1365-1372. [CrossRef] [PubMed]

46. Dean, C.R.; Young, A.F.; Meric, I.; Lee, C.; Wang, L.; Sorgenfrei, S.; Watanabe, K.; Taniguchi, T.; Kim, P.; Shepard, K.L. Boron nitride substrates for high-quality graphene electronics. Nat. Nanotechnol. 2010, 5, 722. [CrossRef] [PubMed]

47. Splendiani, A.; Sun, L.; Zhang, Y.; Li, T.; Kim, J.; Chim, C.-Y.; Galli, G.; Wang, F. Emerging photoluminescence in monolayer $\mathrm{MoS}_{2}$. Nano Lett. 2010, 10, 1271-1275. [CrossRef] [PubMed]

48. Liu, Y.; Yu, D.; Zeng, C.; Miao, Z.; Dai, L. Biocompatible graphene oxide-based glucose biosensors. Langmuir 2010, 26, 6158-6160. [CrossRef] [PubMed]

49. Li, N.; Zhang, Q.; Gao, S.; Song, Q.; Huang, R.; Wang, L.; Liu, L.; Dai, J.; Tang, M.; Cheng, G. Three-dimensional graphene foam as a biocompatible and conductive scaffold for neural stem cells. Sci. Rep. 2013, 3, 1604. [CrossRef] [PubMed] 
50. Weng, Q.; Wang, B.; Wang, X.; Hanagata, N.; Li, X.; Liu, D.; Wang, X.; Jiang, X.; Bando, Y.; Golberg, D. Highly water-soluble, porous, and biocompatible boron nitrides for anticancer drug delivery. ACS Nano 2014, 8 , 6123-6130. [CrossRef] [PubMed]

51. Zhu, Y.; Murali, S.; Cai, W.; Li, X.; Suk, J.W.; Potts, J.R.; Ruoff, R.S. Graphene and graphene oxide: Synthesis, properties, and applications. Adv. Mater. 2010, 22, 3906-3924. [CrossRef] [PubMed]

52. Li, X.; Zhu, H. Two-dimensional $\mathrm{MoS}_{2}$ : Properties, preparation, and applications. J. Mater. 2015, 1, $33-44$. [CrossRef]

53. Zhang, K.; Feng, Y.; Wang, F.; Yang, Z.; Wang, J. Two dimensional hexagonal boron nitride (2D-hBN): Synthesis, properties and applications. J. Mater. Chem. C 2017, 5, 11992-12022. [CrossRef]

54. Fan, M.; Andrade, G.F.; Brolo, A.G. A review on the fabrication of substrates for surface enhanced Raman spectroscopy and their applications in analytical chemistry. Anal. Chim. Acta 2011,693, 7-25. [CrossRef] [PubMed]

55. Brown, R.J.; Milton, M.J. Nanostructures and nanostructured substrates for surface-Enhanced Raman scattering (SERS). J. Raman Spectrosc. 2008, 39, 1313-1326. [CrossRef]

56. Ouyang, L.; Ren, W.; Zhu, L.; Irudayaraj, J. Prosperity to challenges: Recent approaches in SERS substrate fabrication. Rev. Anal. Chem. 2017, 36, 20160027. [CrossRef]

57. Xia, M.; Li, B.; Yin, K.; Capellini, G.; Niu, G.; Gong, Y.; Zhou, W.; Ajayan, P.M.; Xie, Y.-H. Spectroscopic signatures of $\mathrm{AA}^{\prime}$ and $\mathrm{AB}$ stacking of chemical vapor deposited bilayer $\mathrm{MoS}_{2}$. ACS Nano 2015, 9, 12246-12254. [CrossRef] [PubMed]

58. Zhu, X.; Shi, L.; Schmidt, M.S.; Boisen, A.; Hansen, O.; Zi, J.; Xiao, S.; Mortensen, N.A. Enhanced light-matter interactions in graphene-covered gold nanovoid arrays. Nano Lett. 2013, 13, 4690-4696. [CrossRef] [PubMed]

59. Li, H.; Contryman, A.W.; Qian, X.; Ardakani, S.M.; Gong, Y.; Wang, X.; Weisse, J.M.; Lee, C.H.; Zhao, J.; Ajayan, P.M. Optoelectronic crystal of artificial atoms in strain-textured molybdenum disulphide. Nat. Commun. 2015, 6, 7381. [CrossRef] [PubMed]

60. Pirkle, A.; Chan, J.; Venugopal, A.; Hinojos, D.; Magnuson, C.; McDonnell, S.; Colombo, L.; Vogel, E.; Ruoff, R.; Wallace, R. The effect of chemical residues on the physical and electrical properties of chemical vapor deposited graphene transferred to $\mathrm{SiO}_{2}$. Appl. Phys. Lett. 2011, 99, 122108. [CrossRef]

61. Kim, Y.-K.; Han, S.W.; Min, D.-H. Graphene oxide sheath on Ag nanoparticle/graphene hybrid films as an antioxidative coating and enhancer of surface-enhanced Raman scattering. ACS Appl. Mater. Interfaces 2012, 4, 6545-6551. [CrossRef] [PubMed]

62. Hu, Y.; Li, F.; Bai, X.; Li, D.; Hua, S.; Wang, K.; Niu, L. Label-free electrochemical impedance sensing of DNA hybridization based on functionalized graphene sheets. Chem. Commun. 2011, 47, 1743-1745. [CrossRef] [PubMed]

63. Georgakilas, V.; Otyepka, M.; Bourlinos, A.B.; Chandra, V.; Kim, N.; Kemp, K.C.; Hobza, P.; Zboril, R.; Kim, K.S. Functionalization of graphene: Covalent and non-covalent approaches, derivatives and applications. Chem. Rev. 2012, 112, 6156-6214. [CrossRef] [PubMed]

64. Shan, C.; Yang, H.; Han, D.; Zhang, Q.; Ivaska, A.; Niu, L. Water-soluble graphene covalently functionalized by biocompatible poly-L-lysine. Langmuir 2009, 25, 12030-12033. [CrossRef] [PubMed]

65. Liu, Y.; Hu, Y.; Zhang, J. Few-layer graphene-encapsulated metal nanoparticles for surface-enhanced Raman spectroscopy. J. Phys. Chem. C 2014, 118, 8993-8998. [CrossRef]

66. Wei, D.; Lu, Y.; Han, C.; Niu, T.; Chen, W.; Wee, A.T.S. Critical crystal growth of graphene on dielectric substrates at low temperature for electronic devices. Angew. Chem. Int. Ed. 2013, 52, 14121-14126. [CrossRef] [PubMed]

67. Qi, J.; Zheng, W.; Zheng, X.; Wang, X.; Tian, H. Relatively low temperature synthesis of graphene by radio frequency plasma enhanced chemical vapor deposition. Appl. Surf. Sci. 2011, 257, 6531-6534. [CrossRef]

68. Sun, J.; Chen, Y.; Cai, X.; Ma, B.; Chen, Z.; Priydarshi, M.K.; Chen, K.; Gao, T.; Song, X.; Ji, Q. Direct low-temperature synthesis of graphene on various glasses by plasma-enhanced chemical vapor deposition for versatile, cost-effective electrodes. Nano Res. 2015, 8, 3496-3504. [CrossRef]

69. Scarabelli, L.; Coronado-Puchau, M.; Giner-Casares, J.J.; Langer, J.; Liz-Marzán, L.M. Monodisperse gold nanotriangles: Size control, large-scale self-assembly, and performance in surface-enhanced Raman scattering. ACS Nano 2014, 8, 5833-5842. [CrossRef] [PubMed]

70. McLellan, J.M.; Li, Z.-Y.; Siekkinen, A.R.; Xia, Y. The SERS activity of a supported Ag nanocube strongly depends on its orientation relative to laser polarization. Nano Lett. 2007, 7, 1013-1017. [CrossRef] [PubMed] 
71. Nie, S.; Emory, S.R. Probing single molecules and single nanoparticles by surface-enhanced Raman scattering. Science 1997, 275, 1102-1106. [CrossRef] [PubMed]

72. Kha, N.M.; Chen, C.-H.; Su, W.-N.; Rick, J.; Hwang, B.-J. Improved Raman and photoluminescence sensitivity achieved using bifunctional Ag@SiO 2 nanocubes. Phys. Chem. Chem. Phys. 2015, 17, 21226-21235. [CrossRef] [PubMed]

73. Sakano, T.; Tanaka, Y.; Nishimura, R.; Nedyalkov, N.N.; Atanasov, P.A.; Saiki, T.; Obara, M. Surface enhanced raman scattering properties using Au-coated $\mathrm{ZnO}$ nanorods grown by two-step, off-axis pulsed laser deposition. J. Phys. D Appl. Phys. 2008, 41, 235304. [CrossRef]

74. Kim, G.; Kim, M.; Hyun, C.; Hong, S.; Ma, K.Y.; Shin, H.S.; Lim, H. Hexagonal boron nitride/au substrate for manipulating surface plasmon and enhancing capability of surface-enhanced Raman spectroscopy. ACS Nano 2016, 10, 11156-11162. [CrossRef] [PubMed]

75. Liu, Z.; Gong, Y.; Zhou, W.; Ma, L.; Yu, J.; Idrobo, J.C.; Jung, J.; MacDonald, A.H.; Vajtai, R.; Lou, J. Ultrathin high-temperature oxidation-resistant coatings of hexagonal boron nitride. Nat. Commun. 2013, 4, 2541. [CrossRef] [PubMed]

76. Chen, S.; Brown, L.; Levendorf, M.; Cai, W.; Ju, S.-Y.; Edgeworth, J.; Li, X.; Magnuson, C.W.; Velamakanni, A.; Piner, R.D. Oxidation resistance of graphene-coated $\mathrm{Cu}$ and $\mathrm{Cu} / \mathrm{Ni}$ alloy. ACS Nano 2011, 5, 1321-1327. [CrossRef] [PubMed]

77. Liu, X.; Wang, J.; Wu, Y.; Fan, T.; Xu, Y.; Tang, L.; Ying, Y. Compact shielding of graphene monolayer leads to extraordinary SERS-active substrate with large-area uniformity and long-term stability. Sci. Rep. 2015, 5, 17167. [CrossRef] [PubMed]

78. Hu, Y.; Kumar, P.; Xuan, Y.; Deng, B.; Qi, M.; Cheng, G.J. Controlled and stabilized light-matter interaction in graphene: Plasmonic film with large-scale 10-nm lithography. Adv. Opt. Mater. 2016, 4, 1811-1823. [CrossRef]

79. Li, X.; Li, J.; Zhou, X.; Ma, Y.; Zheng, Z.; Duan, X.; Qu, Y. Silver nanoparticles protected by monolayer graphene as a stabilized substrate for surface enhanced Raman spectroscopy. Carbon 2014, 66, 713-719. [CrossRef]

80. Du, Y.; Zhao, Y.; Qu, Y.; Chen, C.-H.; Chen, C.-M.; Chuang, C.-H.; Zhu, Y. Enhanced light-matter interaction of graphene-gold nanoparticle hybrid films for high-performance SERS detection. J. Mater. Chem. C 2014, 2, 4683-4691. [CrossRef]

81. Zhao, Y.; Xie, Y.; Bao, Z.; Tsang, Y.H.; Xie, L.; Chai, Y. Enhanced SERS stability of R6G molecules with monolayer graphene. J. Phys. Chem. C 2014, 118, 11827-11832. [CrossRef]

82. Xie, L.; Ling, X.; Fang, Y.; Zhang, J.; Liu, Z. Graphene as a substrate to suppress fluorescence in resonance Raman spectroscopy. J. Am. Chem. Soc. 2009, 131, 9890-9891. [CrossRef] [PubMed] 